\title{
Thermally Induced Diffusion and Restructuring of Iron Triade (Fe, Co, Ni) Nanoparticles Passivated by Several Layers of Gold
}

\author{
Martin Schnedlitz, Daniel Knez, Maximilian Lasserus, Ferdinand Hofer, Ricardo Fernández-Perea, \\ Andreas W. Hauser,* María Pilar de Lara-Castells,* and Wolfgang E. Ernst*
}

Cite This: J. Phys. Chem. C 2020, 124, 16680-16688

Read Online

\section{ACCESS | Llll Metrics \& More | 国 Article Recommendations | Sl Supporting Information}

ABSTRACT: The temperature-induced structural changes of $\mathrm{Fe}-$, $\mathrm{Co}-$, and $\mathrm{Ni}-\mathrm{Au}$ core-shell nanoparticles with diameters around $5 \mathrm{~nm}$ are studied via atomically resolved transmission electron microscopy. We observe structural transitions from local toward global energy minima induced by elevated temperatures. The experimental observations are accompanied by a computational modeling of all core-shell particles with either centralized or decentralized core positions. The embedded atom model is employed and further supported by density functional theory calculations. We provide a detailed comparison of vacancy formation energies obtained for all materials involved in order to explain the variations in the restructuring processes which we observe in temperature-programmed TEM studies of the particles.
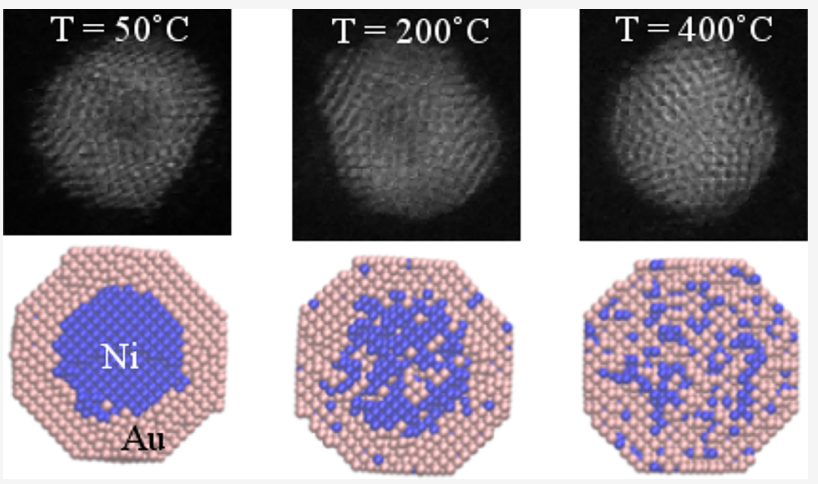

\section{INTRODUCTION}

Bimetallic systems at the nanoscale have recently received increased attention as the combination of intermetallic interactions and surface size effects can trigger unexpected physical behavior and new phenomena. Potential applications cover a wide range of different fields, including biomedical applications, ${ }^{1-5}$ optics, $^{6-9}$ heterogeneous catalysis, ${ }^{10-13}$ electrochemistry, ${ }^{14}$ and electronics. ${ }^{14,15}$ Additionally, magnetic core nanoparticles have been suggested for the activation of stem cells, ${ }^{16,17}$ as enhancers of supercapacitors, ${ }^{18}$ or for the optomagnetic fine-tuning of semiconductors. ${ }^{19}$

Due to their synergistic effects, bimetallic nanoparticles formed by a combination of magnetic and noble metals are particularly interesting for various physicochemical applications such as bifunctional catalysis. ${ }^{20,21}$ Understanding the features of structural stability and metastability in local energetic minima within this novel class of materials would provide us with new possibilities in material design but necessitates an adequate modeling of metallic interactions in large, yet finite systems where surface and interface effects play an equally important role as atomic diffusion. ${ }^{22,23}$ Metastable off-equilibrium structures are particularly strongly affected by thermal rearrangement processes such as surface diffusion, which makes them very interesting for the investigation of thermodynamically induced structural changes but renders their behavior also rather challenging to predict. Recently, the phenomenon of metastability in metallic nanowires has been addressed via cellular automata and molecular dynamics simulations on a coarse grid. ${ }^{24,25}$ However, diffusion processes in mixed-metallic structures cannot be treated on such a macroscopic level due to their intrinsic atomistic character. In this article, our experimental findings are therefore accompanied by atomic structure calculations, followed by a detailed investigation of vacancy formation energies in order to explain the striking structural differences observed in the experiment. In recent articles we have discussed the alloying of silver-gold nanoparticles, ${ }^{26}$ the oxygen-induced rearrangement of centralized core-shell $\mathrm{Fe}-\mathrm{Au}$ nanoparticles, ${ }^{27}$ the oxidation of $\mathrm{Ni}$-Au nanoparticles, ${ }^{28}$ as well as the core location-dependent diffusion dynamics of $\mathrm{Ni}$-Au nanoparticles at elevated temperatures. $^{29}$ The current extension of our ongoing work on bimetallic structures now focuses on the metastability of centralized magnetic cores embedded in a protective $\mathrm{Au}$ shell. Although $\mathrm{Fe}, \mathrm{Co}$, and $\mathrm{Ni}$ are rather similar to each other in their interaction energies as well as their bond lengths, the slight deviations result in a substantially different thermodynamic behavior.

This article is structured as follows. First, the experimental observations of restructuring of $\mathrm{Fe} / \mathrm{Co} / \mathrm{Ni}-\mathrm{Au}$ core-shell clusters at elevated temperatures are presented. Then, vacancy formation energies are calculated from a force field

Received: May 20, 2020

Revised: July 9, 2020

Published: July 9, 2020 
approximation in order to gain insights into the various energy barriers which determine the processes of intermixing or structural rearrangement. For the sake of reliability, parts of our results are benchmarked against periodic electronic structure calculations employing density functional theory. Finally, we investigate the impact of temperature and entropy on the onset of restructuring via large-scale static calculations on a mixed-metallic system containing 6266 atoms at various levels of intermixing.

\section{MATERIAL AND METHODS}

2.1. Nanoparticle Synthesis. The $\mathrm{Fe} / \mathrm{Co} / \mathrm{Ni}-\mathrm{Au}$ coreshell particles are synthesized inside of droplets of superfluid helium, generated in a molecular beam setup which employs an adiabatic expansion of cooled helium into vacuum. The produced nanodroplets serve as "nanolabs" which can be doped with the desired metals by a sequential pickup of metal atoms from the gas phase. A short summary of the process is provided below; for a more elaborate description of our setup we recommend reading of our previous publications on this subject. $^{30,31}$

High-purity $\mathrm{He}(99.9999 \%)$ is expanded through a $5 \mu \mathrm{m}$ nozzle at $7 \mathrm{~K}$ with a pressure of 20 bar into vacuum. A supersonic expansion process leads to the formation of $\mathrm{He}$ clusters with an average number of $10^{7} \mathrm{He}$ atoms and an internal temperature of $0.38 \mathrm{~K}$, which are widely referred to as $\mathrm{He}$ droplets due to their superfluid character. ${ }^{32}$ After the formation, the droplets are exposed to a sequence of metal vapors generated by resistively heated "pickup" cells. In the current setup, the first cell contains either $\mathrm{Fe}, \mathrm{Co}$, or $\mathrm{Ni}$, while the second cell is filled with Au. As a result, spherical Fe/Co/ $\mathrm{Ni}$ core clusters are formed in the droplet which then get surrounded by a protective layer of Au. Although rather laborious and limited in the amount of produced material, this technique allows the convenient synthesis of any combination of core-shell or even onion-type structure. Particle sizes and stoichiometric ratios can be adjusted by simply varying the individual vapor pressures in the cells. Note, however, the stochastic character of the pickup process, which leads to an inevitable log-normal distribution of cluster sizes. For this experiment, an average diameter of $d=6 \pm 2 \mathrm{~nm}$ and a Fe/ $\mathrm{Co} / \mathrm{Ni}: \mathrm{Au}$ ratio of $3: 7$ have been chosen in order to obtain perfect conditions for a complete coating of the $\mathrm{Fe} / \mathrm{Co} / \mathrm{Ni}$ core with a protective layer of $\mathrm{Au}$. The release of binding energy during metal cluster formation results in the partial evaporation of the droplet. This loss of $\mathrm{He}$ can be measured via a residual gas analyzer (Balzer QMA 200/QME 200) and allows the monitoring of the core-shell ratio. The doped He droplet beam is finally terminated on a heatable TEM grid (DENSsolutions Nano-Chip XT SiN). The clusters are deposited on suitable supports in a soft-landing process without significant structural changes, while the excess helium is vaporized upon impact. ${ }^{33-36}$

2.2. Electron Microscopy Characterization. A probecorrected FEI Titan ${ }^{3}$ G2 60-300 is used to record high-angle annular dark-field (HAADF) images of the temperatureinduced restructuring processes in situ. Elemental analysis is provided by a four-quadrant EDX spectroscopy detector and a Gatan Quantum energy filter for electron energy loss spectroscopy (EELS). The in situ heating experiments are carried out in a DENSsolutions Wildfire D5 holder.

2.3. Computational Modeling. Our experimental efforts are accompanied by computational studies employing the
LAMMPS (Large-scale Atomic/Molecular Massively Parallel Simulation) package. ${ }^{37}$ All pairwise intermetallic interactions $(\mathrm{Fe} / \mathrm{Co} / \mathrm{Ni}-\mathrm{Au})$ used in our theoretical studies are based on the embedded atom method (EAM). ${ }^{38}$ It includes the pair energy between atoms $i$ and $j$ as well as contributions due to the embedding of each metal atom $i$ into its local environment described by the electron density $\rho_{i}$. Therefore, it is expected to be more suitable for metal-compound formation as well as for the treatment of surface effects than other pairwise interatomic potentials. In order to provide estimates of the vacancy energies, we employ a periodic setup with supercells comprising $10 \times 10 \times 10$ face-centered-cubic (fcc) unit cells to investigate intermetallic diffusion in the bulk and $10 \times 10 \times 30$ fcc unit cells to study surface energies (i.e., including an extra layer of vacuum). The grids are relaxed before and after the formation of a vacancy with an energy threshold of $10^{-10} \mathrm{eV}$.

Periodic electronic structure calculations are performed with the Vienna Ab initio Simulation Package (VASP 5.4.4), ${ }^{39,40}$ following a similar computational approach to that reported in previous work on $\mathrm{Ag}_{5}-$ and $\mathrm{Cu}_{5}-\mathrm{TiO}_{2}(110)$ interactions. ${ }^{41,42}$ Specifically, we employ a dispersion-corrected DFT-D3 ansatz, $^{43,44}$ given its excellent performance in describing the adsorption of subnanometer silver and copper clusters on oxide surfaces. ${ }^{41,42,45}$ Structural optimizations and the calculation of interaction energies are carried out with the Perdew-Burke-Ernzerhof (PBE) density functional ${ }^{46}$ and the Becke-Johnson (BJ) damping ${ }^{43}$ for the D3 dispersion correction. Minor energy differences and the same trends were found when replacing the PBE functional by the PBEsol $^{47,48}$ counterpart in the DFT-D3 ansatz. The bulk of the considered materials is modeled by employing supercells comprising $5 \times 5 \times 5$ and $4 \times 4 \times 4 \mathrm{fcc}$ unit cells of 500 and 256 atoms, respectively. The surface of the materials is simulated through the introduction of a vacuum region 4 times larger than the width of the $4 \times 4 \times 4$ supercell, which effectively decouples the latter from interactions with periodic images in the direction perpendicular to the surface. Electronion interactions are described by the projector augmentedwave method, ${ }^{40,49}$ using PAW-PBE pseudopotentials as implemented in the program. The electrons of the $\mathrm{Fe}(3 \mathrm{~d}$, $4 s), \mathrm{Co}(3 \mathrm{~d}, 4 \mathrm{~s}), \mathrm{Ni}(3 \mathrm{~d}, 4 \mathrm{~s})$, and $\mathrm{Au}(5 \mathrm{~d}, 6 \mathrm{~s})$ are treated explicitly as valence electrons. A plane-wave basis set with a kinetic energy cutoff of $270 \mathrm{eV}$ is used. The first-order Methfessel and Paxton scheme is employed to account for partial orbital occupations, with a smearing width of $0.2-0.5$ (0.2) $\mathrm{eV}$ in energy calculations of the bulk (surface) of the considered materials. Within a testing window of the smearing width ranging from 0.2 to $0.5 \mathrm{eV}$, the vacancy formation energies in bulk vary by $2 \%$ only. The Brillouin zone is sampled at the $\Gamma$ point. Relative energies using a $2 \times 2 \times 2$ MonkhorstPack $^{50} k$-point mesh differed by less than $0.1 \mathrm{eV}$ from those calculated at the $\Gamma$ point. The convergence criterion is fixed to values from $10^{-4}$ to $10^{-6} \mathrm{eV}$ for the self-consistent electronic minimization. All the atoms from the supercells are relaxed with a force threshold of $0.02 \mathrm{eV} / \AA$. All calculations are spinpolarized.

The impact of intermixing on the Helmholtz free energy for the $\mathrm{Ni}-\mathrm{Au}$ as well as the $\mathrm{Co}-\mathrm{Au}$ system is determined by comparing a centralized with a decentralized core-shell fcc truncated octahedron containing 6266 atoms. Notice that the $\alpha$ bcc phase is the most stable in the bulk of iron for temperatures below ca. $900{ }^{\circ} \mathrm{C}$ (see, e.g., ref 51.) In contrast, the experiment shows that an fcc structure is favored for the 

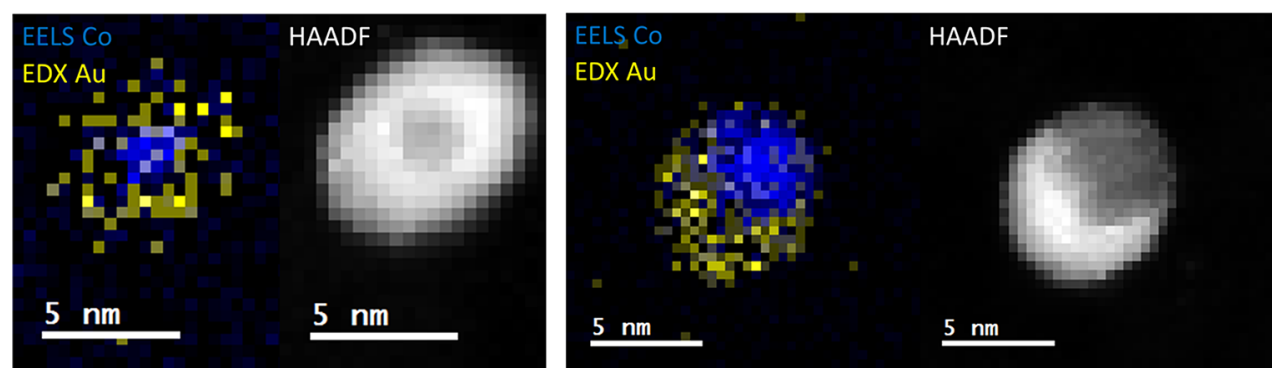

Figure 1. EELS Co observations and EDX Au observations as well as complementary HAADF scans of temperature-induced structural transition from centralized (left) to decentralized (right) $\mathrm{Co}-\mathrm{Au}$ clusters. The temperatures correspond to $25^{\circ} \mathrm{C}$ (left) and $400{ }^{\circ} \mathrm{C}$ (right).

iron core of the $\mathrm{Fe}-\mathrm{Au}$ core-shell nanoparticles synthesized in this work. A core:shell ratio of $3: 7$ is chosen for the simulations, in accordance with the approximate mixing ratios in the experimental setup. The exchange of core and shell atoms is performed in steps of 50 atoms. After each step, the cluster is allowed to fully relax in order to obtain its minimum energy, and the total energy as a function of the number of exchanges is obtained. The number of particle exchanges can be further related to the entropy of the system. This way, estimates of the Helmholtz free energy at various temperatures are obtained, which can be used to study the onset of metallic intermixing.

\section{RESULTS AND DISCUSSION}

We start with the presentation of our experimental findings regarding the structural behavior of the core-shell clusters as observed during temperature-programmed treatments.

3.1. Experimental Observations. Initially, all bimetallic clusters feature a fully centralized core-shell structure due to their identical synthesis as described in Section 2. With a $\mathrm{He}$ nozzle temperature of $7 \mathrm{~K}$ and an approximate metal gas vapor pressure between $10^{-6}$ and $10^{-5}$ mbar, an average particle diameter of $6 \pm 2 \mathrm{~nm}$ is obtained for all three core-shell systems. Overview TEM images before any heat treatment and particle size distributions of all three bimetallic systems are provided in the Supporting Information.

A surface coverage of $1.1 \pm 0.2 \%$ was chosen in order to avoid contact between individual clusters on the substrate. The temperature of the latter was increased in steps of $50{ }^{\circ} \mathrm{C}$, starting at room temperature, and HAADF images were taken at each step. To guarantee an oxygen-free restructuring process, EELS measurements are performed as well in order to check for traces of oxygen. In the following, the TEM observations of the three bimetallic systems are described in detail.

3.1.1. Co-Au. Starting from a centralized core-shell configuration, $\mathrm{Co}-\mathrm{Au}$ clusters undergo a transition toward a decentralized Co core underneath a single protective layer of $\mathrm{Au}$ at $400^{\circ} \mathrm{C}$. At this temperature, the surface mobility is high enough to enable the cluster to undergo a transition toward an energetically preferred configuration. Their structural evolution with increasing temperature is illustrated in Figure S2 of the Supporting Information, providing TEM images taken at steps of $100{ }^{\circ} \mathrm{C}$.

This transition to a decentralized Co core has been predicted by Palomares et al., ${ }^{52}$ having its origin in the reduced overall stress inside the cluster, which is caused by the lattice mismatch between layers of $\mathrm{Co}$ and $\mathrm{Au}$. In Figure 1, EELS and EDX elemental maps and HAADF recordings illustrate the transition from a centralized cluster toward a decentralized cluster at $400{ }^{\circ} \mathrm{C}$. The Co core remains intact throughout the whole solid regime, without any tendency toward alloying. This configuration is stable up to melting temperature $\left(1100^{\circ} \mathrm{C}\right)$. As soon as melting starts, the decentralized cluster undergoes a transition toward the mixed phase.

3.1.2. $\mathrm{Fe}-\mathrm{Au}$. The $\mathrm{Fe}-\mathrm{Au}$ system behaves similarly to the $\mathrm{Co}-\mathrm{Au}$ system in its preference for a decentralization of the core as soon as atomic mobility becomes high enough. However, the process of decentralization is rather different from the previous: At a temperature of $400{ }^{\circ} \mathrm{C}$, the centralized Fe core fractures into several decentralized Fe pieces, but these fragments remain underneath one protective layer of $\mathrm{Au}$ (see Figure 2). This remarkable behavior makes the $\mathrm{Fe}-\mathrm{Au}$
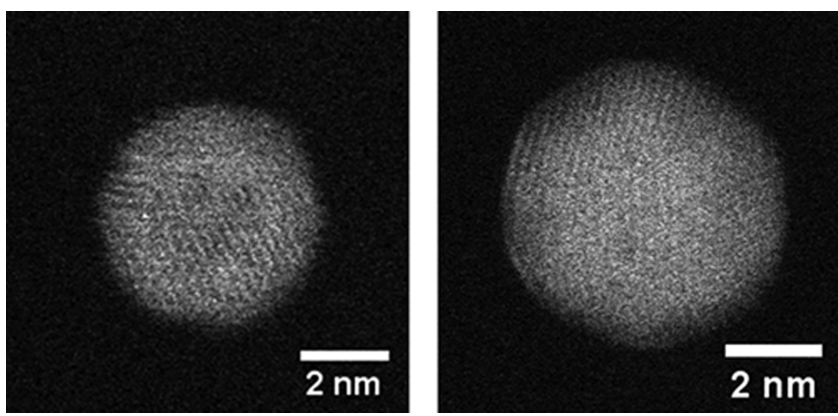

Figure 2. HAADF observation of temperature-induced rearrangements within a $\mathrm{Fe}-\mathrm{Au}$ cluster. At $25^{\circ} \mathrm{C}$ the cluster remains in its centralized state (left), whereas a temperature increase to $400{ }^{\circ} \mathrm{C}$ results in the rearrangement toward a fractured $\mathrm{Fe}$ core located underneath the Au surface.

combination stand out among the three systems of this study. However, similar effects of an energetically preferred ringlike aggregation of material near the surface have been predicted for related systems (e.g., the $\mathrm{Co} / \mathrm{Ni}-\mathrm{Ag}$ system). ${ }^{53}$ The structural changes in the $\mathrm{Fe}-\mathrm{Au}$ system are documented in Figures S4 and S5 of the Supporting Information, which contain TEM pictures taken at room temperature 500 and $1100^{\circ} \mathrm{C}$.

3.1.3. $\mathrm{Ni}-\mathrm{Au}$. In contrast to the decentralization tendencies observed for $\mathrm{Co}-\mathrm{Au}$ and $\mathrm{Fe}-\mathrm{Au}$, the $\mathrm{Ni}-\mathrm{Au}$ system undergoes a phase transition toward full alloying even before reaching the melting temperature (see Figure 3 ). The temperature regime for this transition seems to overlap with that corresponding to a transition toward a decentralized $\mathrm{Ni}$ core. The structural changes are documented by a series of TEM images in Figure S7 of the Supporting Information. The latter structure has been found to be more stable. ${ }^{29}$ Note that the formation of a Ni-Au alloy is in agreement with the phase 

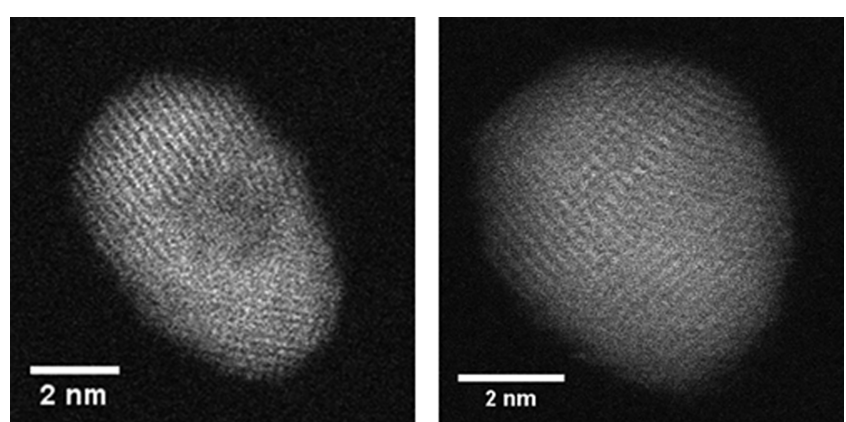

Figure 3. HAADF observation of temperature-induced rearrangements within a $\mathrm{Ni}-\mathrm{Au}$ cluster. The cluster is embedded centrally inside a $\mathrm{Au}$ shell. At $300-400{ }^{\circ} \mathrm{C}$ the $\mathrm{Ni}$ and $\mathrm{Au}$ atoms start to form an alloy, still exhibiting a lattice structure.

diagram for the bulk material, which predicts an alloyed solid phase at temperatures above $300^{\circ} \mathrm{C}$. A transition from a centralized cluster toward a decentralized cluster at temperatures below the alloying threshold of $300-400{ }^{\circ} \mathrm{C}$ can only be observed if excess energy is introduced to the system via electron beam damage (see ref 54). We note further that an oxidation of $\mathrm{Ni}$ atoms can trigger core-shell inversions, as has been demonstrated in a recent article. ${ }^{27}$ Therefore, the chosen heating ramps have to be fast enough to prevent any potential oxidation stemming from surface water presence below $400{ }^{\circ} \mathrm{C}$.

3.1.4. Similarities between the Systems. Although in different ways, all three systems undergo a transition from their initial centralized core-shell arrangement toward an energetically more stable configuration at temperatures around $400{ }^{\circ} \mathrm{C}$. Furthermore, it is found that for all three types of bimetallic nanoclusters the bulk phase diagram is correctly predicting whether alloying can occur or not. However, the actual temperature for such a phase transition is lower than predicted due to surface effects. This experimentally observed diversity of the various restructuring processes as described above is most likely related to differences in surface mobilities and interfacial energies.

3.2. Diffusion and Intermixing via Vacancy Formation. As diffusion and intermixing are driven by the formation of vacancies in general, the energy necessary to form vacancies, $E_{\mathrm{f}}$, is a key element to understand these differences in the experimental observations. An estimation of $E_{\mathrm{f}}$ is obtained from force field calculations with the LAMMPS program package as described in Section 2. $E_{\mathrm{f}}$ is obtained by comparing the energy of the full periodic supercell before $\left(E_{\mathrm{b}}\right)$ and after $\left(E_{\mathrm{v}}\right)$ removal of one of its $\mathrm{N}$ atoms, according to ${ }^{55}$

$$
E_{\mathrm{f}}=E_{\mathrm{v}}-\frac{N-1}{N} E_{\mathrm{b}}
$$

The force field contains interaction energies only; therefore, cohesive energies $E_{c}$ can be obtained by simply dividing the total energy of the slab by the number of atoms. Our results are summarized in Table 1 . According to ref 56, vacancy formation energies are approximately proportional to their corresponding cohesive energies $E_{\mathcal{c}}$ as expressed by the empirical relation $E_{\mathrm{f}} /$ $E_{\mathrm{c}}=1 / 3$. However, the $\mathrm{Fe} / \mathrm{Co} / \mathrm{Ni}$ energies in our calculations show a diametrical behavior, with $\mathrm{Ni}$ having the highest $E_{\mathrm{c}}$ and the lowest $E_{\mathrm{f}}$ in comparison to Co and Fe.

Table 1 reveals that $E_{\mathrm{f}}$ as well as $E_{\mathrm{c}}$ are the lowest for Au by far, which indicates that vacancies in gold are formed first upon heating. From this follows that, while the core is still free of vacancies, the shell is already affected by structural changes via
Table 1. Vacancy Formation Energies $E_{\mathrm{f}}$ and Cohesive Energies $E_{c}$ for $\mathrm{Au}, \mathrm{Ni}$, Co, and $\mathrm{Fe}$, Together with the Energy Differences When Replacing an Atom of Type B (Column) in a Lattice of B Atoms with an "Intruding" Atom of Type A (Row)

\begin{tabular}{ccccc}
$E(\mathrm{eV})$ & $\mathrm{Au}$ & $\mathrm{Ni}$ & $\mathrm{Co}$ & $\mathrm{Fe}$ \\
$E_{\mathrm{f}}$ & 1.01 & 1.70 & 1.83 & 1.95 \\
$E_{\mathrm{c}}$ & 3.93 & 4.45 & 4.40 & 4.20 \\
$\mathrm{Au}_{\text {int }}$ & 0.00 & 0.58 & 0.41 & 0.76 \\
$\mathrm{Ni}_{\text {int }}$ & -0.52 & 0.00 & & \\
$\mathrm{Co}_{\text {int }}$ & -0.48 & & 0.00 & \\
$\mathrm{Fe}_{\text {int }}$ & -1.06 & & & 0.00 \\
\hline
\end{tabular}

the migration of vacancies. As soon as vacancies start to appear in the core, the $\mathrm{Au}$ atoms diffuse into the core region as well, leading to the onset of intermixing. When comparing the vacancy energies among the iron triade, it can be seen that $E_{\mathrm{f}}$ is smallest for $\mathrm{Ni}$, followed by $\mathrm{Co}$ and $\mathrm{Fe}$. This energetic comparison implies that $\mathrm{Au}$ can intermix with $\mathrm{Ni}$ at lower temperatures, while higher temperatures are needed for Co and $\mathrm{Fe}$, respectively. Depending on the particle size, the necessary temperature might already exceed the actual melting temperature of the system.

Table 1 also contains the exchange energies for atomic replacements in the bulk, i.e., for the replacement of atom $B$ from a lattice of type B atoms (element in a column) with an atom of type A (element in a row). For clarity, the "intruding" atom is denoted by a subscript "int". When comparing Co and $\mathrm{Fe}$, Co has not only a higher $E_{\mathrm{c}}$ but also a less favorable exchange energy when placed within a lattice of $\mathrm{Au}$ atoms. These findings agree with the experimental observation of a much higher core stability in the case of Co.

3.2.1. Surface Vacancy Formation. Of further interest is the dependence of the vacancy formation energy on the actual position of the vacancy. Obviously, the effort to create such a defect is minimal on the surface. Even at interfaces, the emergence of vacancies is more likely at places with a large lattice mismatch or sufficient "room" between the two metals. Therefore, surface vacancies can be considered as estimates for neutral comparisons between the various systems. We note that an actual calculation of interface energies is possible but extremely tedious due to the many possibilities created by lattice mismatch and the many combinations of Miller indices. Even if available, the final effect would be an average of all of these but mostly dictated by low-energy processes, i.e., those closest to the surface vacancy formation energies.

The determination of the corresponding energy, $E_{\mathrm{f}, \mathrm{s}}$, is carried out in the same manner as mentioned above but with the restriction that the removed element must stem from the surface layer of the material. This energy is then used to calculate the vacancy formation energy according to eq 1 . We use the same number of fcc unit cells as for the studies on intermetallic vacancies but assume periodicity only in the $x$ and $y$ dimension. This is achieved by an extension of the unit cell in the $z$ direction by an additional vacuum layer comprising the volume of $10 \times 10 \times 20 \mathrm{fcc}$ unit cells, resulting in a total supercell size of $10 \times 10 \times 30$ unit cells. The largest relative reduction in comparison to the bulk vacancy, $E_{\mathrm{f}, \mathrm{s}} / E_{\mathrm{f}}$, appears for $\mathrm{Fe}$ (reduced by $76 \%$ ), followed by $\mathrm{Co}$ and $\mathrm{Ni}$ with almost identical ratios (reduced by 66\%) and Au (reduced by 56\%). This pronounced compliance of Fe to accept surface defects and restructuring is particularly interesting in light of the 
experimentally observed core fragmentation which occurs for the $\mathrm{Fe}-\mathrm{Au}$ system only. Together with the results for atomic exchange energies obtained in the previous section, the energy ratios $E_{\mathrm{f}, \mathrm{s}} / E_{\mathrm{f}}$ also provide an explanation as to why the Co core remains intact in the heating experiment and migrates toward a subsurface position.

We further extend this analysis of vacancy formation energies to the two subsurface layers in order to investigate the impact of finiteness also in the underlying layers of a given material. The results are summarized in Table 2. For Ni, Co,

Table 2. Absolute and Relative Vacancy Formation Energies at the Surface and the First Two Subsurface Layers of Au, $\mathrm{Ni}$, Co, and Fe, Calculated at the EAM Level of Theory

\begin{tabular}{lcccc} 
& $\mathrm{Au}$ & $\mathrm{Ni}$ & $\mathrm{Co}$ & $\mathrm{Fe}$ \\
$E_{\mathrm{f}, \mathrm{s}}(\mathrm{eV})$ & 0.44 & 0.57 & 0.61 & 0.47 \\
$E_{\mathrm{f}, \mathrm{s}} / E_{\mathrm{f}}$ & 0.44 & 0.34 & 0.34 & 0.24 \\
$E_{\mathrm{f}, \mathrm{s}-1} / E_{\mathrm{f}}$ & 0.80 & 1.00 & 1.00 & 1.04 \\
$E_{\mathrm{f}, \mathrm{s}-2} / E_{\mathrm{f}}$ & 1.00 & 1.00 & 1.00 & 1.04 \\
\hline
\end{tabular}

and $\mathrm{Fe}$, the creation of vacancies in the first or the second layer below the surface is already fully equivalent to a vacancy creation in the bulk (i.e., the energy differences lie below the accuracy of the method). However, for $\mathrm{Au}$, an intermediate value between bulk and surface energy is obtained for the first subsurface layer, which suggests an increased mobility of vacancies also in the vicinity of surfaces and interfaces. Regarding the vacancy formation energies on the surface, it is interesting to see that the same relative energies $E_{\mathrm{f}, \mathrm{s}} / E_{\mathrm{f}}$ are obtained for $\mathrm{Ni}$ and $\mathrm{Co}$, while an even smaller value is obtained for Fe.

3.2.2. DFT Results for Bulk Vacancies. In this section we provide complementary periodic density functional theory calculations of the vacancy formation energies, starting with the results for the bulk materials, presented in a similar fashion as for the EAM approach discussed above. Note that these results are obtained at a higher level of theory but for smaller $(4 \times 4 \times 4)$ fcc supercells due to the otherwise too high computational effort. Our results are summarized in Table 3.

Table 3. Vacancy Formation $E_{\mathrm{f}}$ Energies for $\mathrm{Au}, \mathrm{Ni}, \mathrm{Co}$, and Fe, Obtained with Density Functional Theory, Evaluated for the Bulk and for the Surface

\begin{tabular}{lcccc}
\multicolumn{1}{c}{$E$} & $\mathrm{Au}$ & $\mathrm{Ni}$ & $\mathrm{Co}$ & $\mathrm{Fe}$ \\
$E_{\mathrm{f}}(\mathrm{eV})$ & 0.67 & 1.63 & 2.26 & 2.02 \\
$E_{\mathrm{f}, \mathrm{s}}(\mathrm{eV})$ & 0.52 & 0.89 & 1.13 & 0.62 \\
$E_{\mathrm{f}, \mathrm{s}} / E_{\mathrm{f}}$ & 0.78 & 0.55 & 0.50 & 0.31 \\
\hline
\end{tabular}

The estimated vacancy formation energies agree well with recently reported DFT-based values. ${ }^{57}$ When compared to our results obtained at the EAM level of theory, we find reasonable agreement for the formation energies of $\mathrm{Fe}, \mathrm{Co}$, and $\mathrm{Ni}$, which are reproduced in the same energetic order and with relative deviations of less than $10 \%$ on average. However, for Au a larger deviation is noticeable. We note that even larger deviations between EAM and DFT occur if the inclusion of dispersion correction in the last steps of the DFT geometry relaxation (which is our standard approach throughout the article) is skipped. A tentative increase to $(5 \times 5 \times 5)$ supercells yields differences in the range of $10 \%$, indicating an acceptable remaining slab size effect.
Atomic exchange energies have been recalculated with DFT as well but are less straightforward to compare since DFT energies are absolute energies, which has to be taken into consideration when taking differences before and after the insertion of atom A in a lattice of atoms B. Using single atom energies obtained with the same method and slab size and employing the formula

$$
\Delta E=E_{\text {AinBslab }}+E_{\text {Batom }}-E_{\text {Bslab }}-E_{\text {Aatom }}
$$

to calculate the exchange energies, the overall tendencies of the EAM table are confirmed, but the values are off by a factor of 3 on average, even if the slab size is increased to $5 \times 5 \times 5$, and differences in the detailed energetic order become apparent. In particular, the insertion of the large $\mathrm{Au}$ atom into a smaller lattice is prone to bias due to the computationally inevitable slab size limitations and is much more problematic than the formation of vacancies in a finite slab of feasible size.

3.2.3. DFT-Based Surface Vacancy Formation Energies. Surface vacancy formation energies have also been calculated at the DFT level. To accelerate the convergence, the geometry relaxation of all atoms from $(4 \times 4 \times 4)$ slabs was carried out with the PBE functional in a first step. Then, the dispersioncorrected DFT scheme was applied on top of the PBE-relaxed structures, and the atoms were allowed to relax further with the exception of cobalt. Our results have been added to Table 3 .

As reported in ref 58 for vacancies at the surfaces of fcc Au and $\mathrm{Ni}$ surfaces [(111), (100), and (110) facets], the vacancy formation energies at the first layer lie below $1 \mathrm{eV}$. Specifically, values between 0.15 [(110) facet] and $0.54 \mathrm{eV}$ [(111) facet] have been reported for iron surfaces, while for nickel values between 0.34 ([110] facet) and $0.82 \mathrm{eV}$ ([111] facet) can be found in the literature. ${ }^{58}$ We notice that the magnitude of the dispersion-free DFT $E_{\mathrm{f}}$ value for the $\mathrm{Fe}(001)$ surface $(0.72 \mathrm{eV})$ is close to that reported in ref $59(0.9 \mathrm{eV})$. For cobalt, the dispersion-corrected vacancy formation energy in the first layer $(1.13 \mathrm{eV})$ is slightly smaller than reported in ref $60(1.4 \mathrm{eV})$.

In agreement with the EAM calculations, the formation of surface vacancies requires much less energy than in the bulk, in particular for Fe. The same holds true when analyzing the dependence of the vacancy formation energy on the layer position for $\mathrm{Au}$ and $\mathrm{Ni}$.

3.3. Computational Intermixing. The comparison of vacancy energies provides only limited information on diffusion tendencies. Therefore, we extend our computational investigation toward the inclusion of entropy effects to obtain estimates of the Helmholtz free energy as a function of the temperature. This way, it should be possible to predict the energetically preferred structural arrangement of each bimetallic nanoparticle at a given temperature in the experiment. At $0 \mathrm{~K}$ we find that a decentralized core is the energetically most favorable configuration for the $\mathrm{Co}-\mathrm{Au}$ and $\mathrm{Ni}-\mathrm{Au}$ systems. However, for $\mathrm{Fe}-\mathrm{Au}$ we do not see a significant energy difference between a centralized and a fully decentralized core, which might be due to a more favorable fractured decentralized core. The impact of temperature is modeled as follows.

Starting from centralized as well as decentralized core-shell structures for the $\mathrm{Fe}-, \mathrm{Co}-$, or $\mathrm{Ni}-\mathrm{Au}$ systems, we perform a series of exchanges between atoms of the core region with $\mathrm{Au}$ atoms of the surrounding Au shell, allowing a stepwise increase of the mixing entropy with each exchange of two atoms. A truncated octahedron fcc cluster, containing 6266 atoms in total and a core:shell atomic ratio of $3: 7$, is adopted in the simulations, which corresponds to $N_{\mathrm{C}}=1880$ core and $N_{\mathrm{S}}=$ 
4386 shell atoms. The exchanges are performed in steps comprising 50 swaps of atomic positions. Each of these steps (50 swaps in the first, 100 in the second, and so on) is repeated 150 times, and the mean value of the energy $E$ is calculated. As an example, the structures of a centralized and decentralized $\mathrm{Co}-\mathrm{Au}$ cluster after the first 50 atomic swaps are depicted in
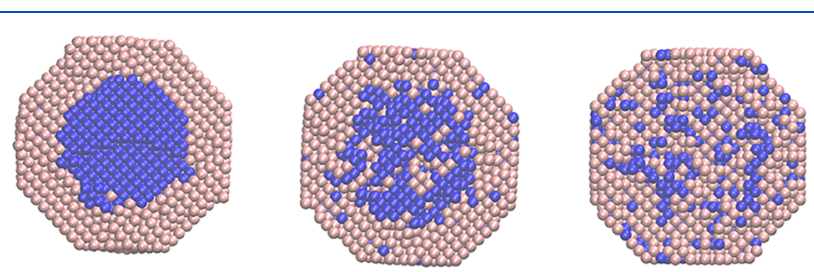

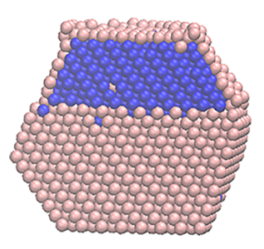

$x=50$

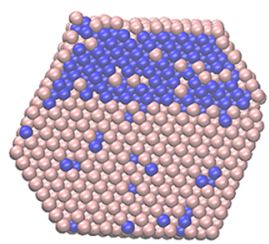

$x=500$

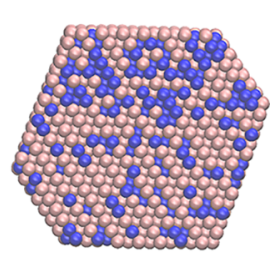

$x=1500$
Figure 4. Cuts through the atomic models of a centralized (upper row) and a decentralized (lower row) $\mathrm{Ni}-\mathrm{Au}$ cluster at different degrees of intermixing $(x=50,500,1500)$.

Figure 4. At each step, the Helmholtz free energy is obtained via

$$
F(x, T)=E(x)-T \cdot S_{\text {mix }}(x)
$$

with $E(x)$ as the inner energy at $0 \mathrm{~K}$ and $S_{\text {mix }}(x)$ as the mixing entropy, both written as a function of the degree of mixing, expressed by $x$, the number of "mobilized" core atoms. With this term we refer to a subgroup of core atoms which are allowed to be placed anywhere in the cluster (including shell as well as core positions, except for those core places occupied by the $N_{\mathrm{C}}-x$ yet "immobilized" core atoms). In each exchange step, $x$ core atoms change places with any of the allowed atoms, followed by a relaxation of the whole system. We obtain the following expression for the mixing entropy as a function of $x$ :

$$
S_{\text {mix }}\left(x, N_{\mathrm{S}}, N_{\mathrm{C}}\right)=k_{\mathrm{B}} \ln \left[\sum_{j=0}^{x}\left(\begin{array}{c}
N_{\mathrm{S}} \\
j
\end{array}\right)\left(\begin{array}{c}
N_{\mathrm{C}} \\
j
\end{array}\right)\right]
$$

In this formula, $j$ denotes the number of core atoms which have swapped their position with shell atoms, which is obviously identical with the number of shell atoms now placed inside the core, and $k_{\mathrm{B}}$ is the Boltzmann constant. Note the difference between $j$ and $x$ in this context: $j$ goes from 0 to $x$ in a summation over products of binomial coefficients, yielding the correct number of possible structures for the current state of intermixing described by $x$. This allows us to calculate the impact of entropy effects for each starting geometry. For $x \rightarrow$ $N_{\mathrm{C}}$, this expression converges toward

$$
S_{\max }\left(N_{\mathrm{C}}, N_{\mathrm{S}}, N_{\mathrm{C}}\right)=k_{\mathrm{B}} \ln \left[\left(\begin{array}{c}
N_{\mathrm{S}}+N_{\mathrm{C}} \\
N_{\mathrm{C}}
\end{array}\right)\right]
$$

which can be proven by Vandermonde's theorem. In this case, the maximum number of possible mixing states is obtained since all intermixing constraints are removed.

Using the expression for $S_{\text {mix }}$ of eq 4 , we can calculate the Helmholtz free energy at various temperatures for all partially intermixed states. We note that our approach is neglecting vibrational contributions to the entropy, but their influence is

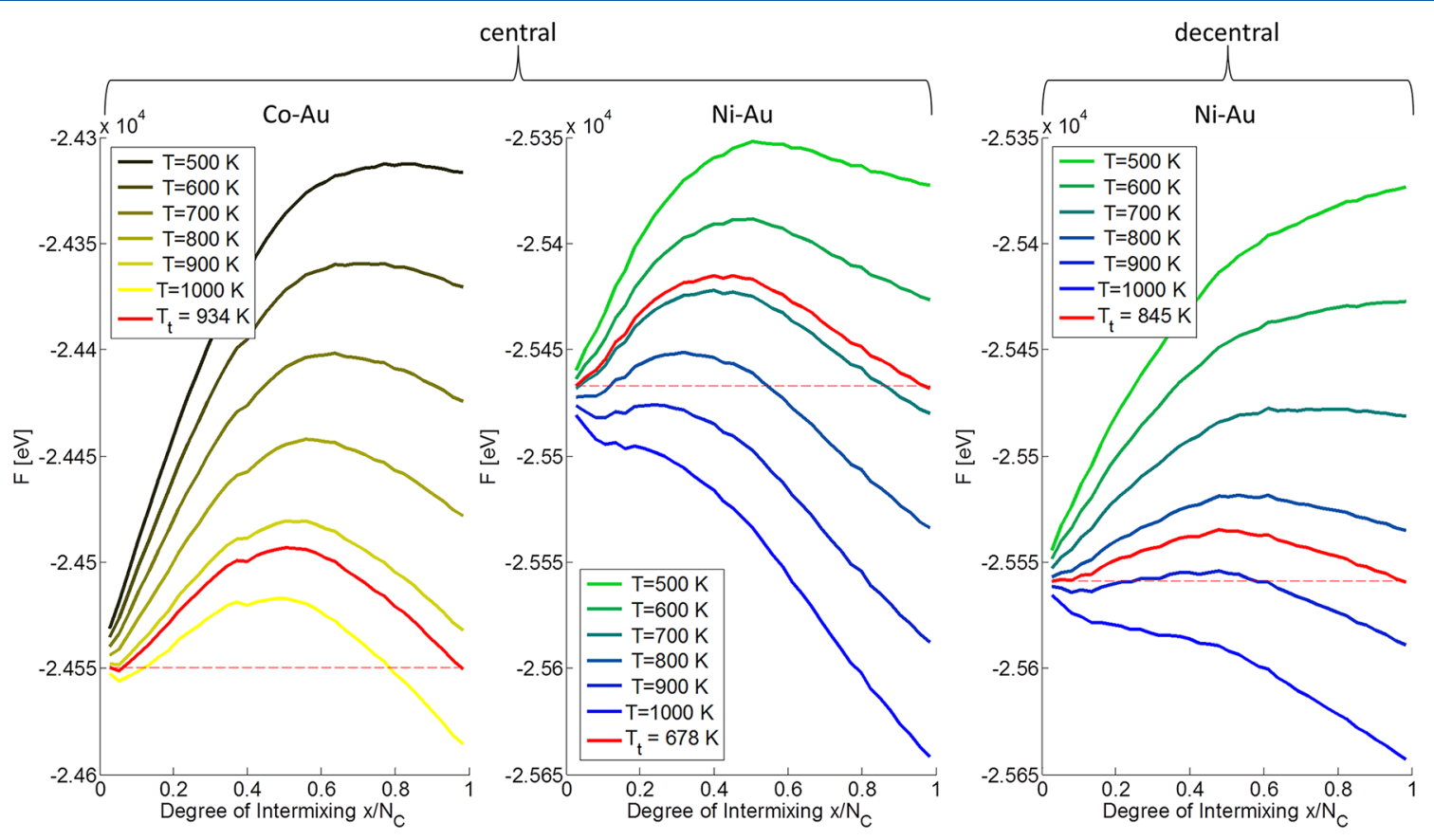

Figure 5. Helmholtz free energies of three bimetallic clusters, plotted as a function of the relative intermixing $x / N_{\mathrm{C}}$. Zero mixing corresponds to a perfect core-shell structure with a minimum of intermetallic bonds. Left: $\mathrm{Co}-\mathrm{Au}$ cluster with centralized $\mathrm{Co}$ core. Center: $\mathrm{Ni}-\mathrm{Au}$ cluster with centralized Ni cluster. Right: $\mathrm{Ni}-\mathrm{Au}$ cluster with decentralized Ni cluster. Intermixed phases become favorable at 914, 662, and 830 K, respectively (red curves). Note the slightly increased resistance against mixing in the case of a decentralized core positioning for Ni-Au. 
known to be marginal. ${ }^{52}$ For graphical illustration, it is most convenient to plot the Helmholtz free energy as a function of $x / N_{\mathrm{C}}$. This way, a relative degree of intermixing is obtained for the $x$ axis, with values between 0 (corresponding to a perfect core-shell structure) and 1 (corresponding to a fully mixed structure). As can be seen in Figure 5, the formation of an alloy in the $\mathrm{Ni}-\mathrm{Au}$ system, starting from a centralized Ni core, becomes feasible at a temperature of $662 \mathrm{~K}$. This value can be derived from the figure by observing the temperature at which the free energy value for an intermixed geometry (right end of each curve) becomes lower than the value for an unmixed core-shell geometry (left end of the curve). This is indicated by a dashed horizontal line for the red curve belonging to the threshold temperature. The value obtained for $\mathrm{Ni}-\mathrm{Au}$ is in good agreement with the experimentally determined intermixing temperature of $570-670 \mathrm{~K}\left(300-400{ }^{\circ} \mathrm{C}\right)$. It indicates that intermixing via $\mathrm{Ni}$ mobility is taking place even in the solid phase, as it is predicted by the bimetallic phase diagram for bulk $\mathrm{Ni}-\mathrm{Au}$. In contrast, a centralized Co core (left panel of Figure 5) is stable up to a temperature of $914 \mathrm{~K}$, which lies above the experimentally observed temperature $(670 \mathrm{~K})$ for core migration from a centralized to a decentralized position. $\mathrm{Co}$ and $\mathrm{Fe}$ do not undergo a transition toward an intermixed cluster, as predicted by their corresponding phase diagrams $\left(\mathrm{Co}-\mathrm{Au},{ }^{61} \mathrm{Fe}-\mathrm{Au}^{62}\right)$, which is due to the energetically more favorable unmixed configuration. Restructuring of the bimetallic cluster is enabled by the increased mobility of surface atoms, as described in Section 3.2. Furthermore, it is found that a decentralized core (right graph in Figure 5) requires higher temperatures for intermixing than a centralized core. This confirms the findings of our previous study ${ }^{29}$ that a decentralized $\mathrm{Ni}$ core requires more energy to form an alloy than a centralized $\mathrm{Ni}$ core. We note that a similar graph for the $\mathrm{Fe}-\mathrm{Au}$ system has been omitted here since its corresponding Helmholtz free energies are fully dominated by the energy contributions from increased intermixing. For the $\mathrm{Fe}-\mathrm{Au}$ potential used in this study, the curves do not show a local minimum at the mixed state within the experimentally relevant range of temperatures.

\section{CONCLUSIONS}

Centralized $\mathrm{Fe}-\mathrm{Au}, \mathrm{Co}-\mathrm{Au}$, and $\mathrm{Ni}-\mathrm{Au}$ core-shell nanoparticles are synthesized in He nanodroplets and deposited on heatable TEM grids. We then study the structural stability of their cores, which are initially fully embedded in a protective outer layer of $\mathrm{Au}$, via transmission electron microscopy while heating the support. We find that even though the three elements of the iron triade are only varying minimally in their respective cohesive energies and bond lengths, entirely different structural behavior is observed upon thermal treatment. The interpretation of our experimental findings is supported by a theoretical comparison of vacancy energies at the EAM and the DFT level, calculated for the four metals, and by studies of Helmholtz free energies of the bimetallic systems at various degrees of intermixing.

The Co core shows a transition toward a decentralized position at $400{ }^{\circ} \mathrm{C}$, which we explain as the result of lattice strain reduction. The $\mathrm{Fe}$ core behaves in a similar way, but instead of remaining a single cohesive mass, it disintegrates into several fragments at $400{ }^{\circ} \mathrm{C}$, also located at decentralized positions. We attribute this behavior to the reduced surface vacancy formation energy of $\mathrm{Fe}$ in comparison to $\mathrm{Co}$. The $\mathrm{Ni}-$ $\mathrm{Au}$ cluster, on the other hand, undergoes a transition toward full intermixing already between 300 and $400{ }^{\circ} \mathrm{C}$. Here, the smaller vacancy formation energy required for the formation of $\mathrm{Ni}$ vacancies enables $\mathrm{Au}$ atoms to migrate into the core at even lower temperatures than in the $\mathrm{Fe}-\mathrm{Au}$ and $\mathrm{Co}-\mathrm{Au}$ systems.

\section{ASSOCIATED CONTENT}

\section{Supporting Information}

The Supporting Information is available free of charge at https://pubs.acs.org/doi/10.1021/acs.jpcc.0c04561.

Additional TEM images of the three bimetallic systems, providing more details regarding the thermally induced structural transitions observed in the experiment (PDF)

\section{AUTHOR INFORMATION}

\section{Corresponding Authors}

Andreas W. Hauser - Institute of Experimental Physics, Graz University of Technology, A-8010 Graz, Austria; 10 orcid.org/ 0000-0001-6918-3106; Email: andreas.w.hauser@gmail.com

María Pilar de Lara-Castells - Institute of Fundamental Physics (AbinitSim Unit), C.S.I.C, E-28006 Madrid, Spain; ○ orcid.org/0000-0001-8697-5770;

Email: Pilar.deLara.Castells@csic.es

Wolfgang E. Ernst - Institute of Experimental Physics, Graz University of Technology, A-8010 Graz, Austria; (1) orcid.org/ 0000-0001-8849-5658; Email: wolfgang.ernst@tugraz.at

\section{Authors}

Martin Schnedlitz - Institute of Experimental Physics, Graz University of Technology, A-8010 Graz, Austria

Daniel Knez - Institute for Electron Microscopy and Nanoanalysis \& Graz Centre for Electron Microscopy, Graz University of Technology, A-8010 Graz, Austria; (1) orcid.org/ 0000-0003-0755-958X

Maximilian Lasserus - Institute of Experimental Physics, Graz University of Technology, A-8010 Graz, Austria

Ferdinand Hofer - Institute for Electron Microscopy and Nanoanalysis \& Graz Centre for Electron Microscopy, Graz University of Technology, A-8010 Graz, Austria

Ricardo Fernández-Perea - Instituto de Estructura de la Materia (C.S.I.C.), E-28006 Madrid, Spain

Complete contact information is available at: https://pubs.acs.org/10.1021/acs.jpcc.0c04561

\section{Notes}

The authors declare no competing financial interest.

\section{ACKNOWLEDGMENTS}

This research has been supported by the Austrian Science Fund (FWF) under Grant No. P 29893-N36, the FWF and the Christian Doppler Research Association (CDG) under Grant No. PIR8-N34, the Horizon 2020 research program of the European Union under Grant No. 823717-ESTEEM3, and the Spanish Agencia Estatal de Investigación (AEI) and the Fondo Europeo de Desarrollo Regional (FEDER, UE) under Grant No. MAT2016-75354-P. The authors acknowledge the use of HPC resources provided by the ZID of Graz University of Technology and by the Vienna Scientific Cluster (VSC). Further support by NAWI Graz is gratefully acknowledged. The CESGA supercomputer center (Spain) is also acknowledged for having provided computational resources. 


\section{REFERENCES}

(1) Wang, P.; Lin, L.; Guo, Z.; Chen, J.; Tian, H.; Chen, X.; Yang, H. Highly Fluorescent Gene Carrier Based on Ag-Au Alloy Nanoclusters. Macromol. Biosci. 2016, 16, 160-167.

(2) Srinoi, P.; Chen, Y.-T.; Vittur, V.; Marquez, M. D.; Lee, T. R. Bimetallic Nanoparticles: Enhanced Magnetic and Optical Properties for Emerging Biological Applications. Appl. Sci. 2018, 8, 1106.

(3) Samanta, A.; Medintz, I. L. Nanoparticles and DNA - a Powerful and Growing Functional Combination in Bionanotechnology. Nanoscale 2016, 8, 9037-9095.

(4) Fan, Z.; Shelton, M.; Singh, A. K.; Senapati, D.; Khan, S. A.; Ray, P. C. Multifunctional Plasmonic Shell-Magnetic Core Nanoparticles for Targeted Diagnostics, Isolation, and Photothermal Destruction of Tumor Cells. ACS Nano 2012, 6, 1065-1073.

(5) Maduraiveeran, G.; Rasik, R.; Sasidharan, M.; Jin, W. Bimetallic Gold-Nickel Nanoparticles as a Sensitive Amperometric Sensing Platform for Acetaminophen in Human Serum. J. Electroanal. Chem. 2018, 808, 259-265.

(6) Jia, X.; Li, J.; Zhang, X.; Wang, E. Controlling the Synthesis and Assembly of Fluorescent $\mathrm{Au} / \mathrm{Ag}$ Alloy Nanoclusters. Chem. Commun. 2015, 51, 17417-17419.

(7) Zhu, J. Surface Plasmon Resonance from Bimetallic Interface in Au-Ag Core-Shell Structure Nanowires. Nanoscale Res. Lett. 2009, 4, 977-981.

(8) Wu, Z.; et al. Assembly-Induced Enhancement of $\mathrm{Cu}$ Nanoclusters Luminescence with Mechanochromic Property. J. Am. Chem. Soc. 2015, 137, 12906-12913.

(9) Bhattacharyya, D.; Sarswat, P. K.; Islam, M.; Kumar, G.; Misra, M.; Free, M. L. Geometrical Modifications and Tuning of Optical and Surface Plasmon Resonance Behaviour of $\mathrm{Au}$ and $\mathrm{Ag}$ Coated $\mathrm{TiO}_{2}$ Nanotubular Arrays. RSC Adv. 2015, 5, 70361-70370.

(10) Wanjala, B. N.; et al. Nanoscale Alloying, Phase-Segregation, and Core-Shell Evolution of Gold-Platinum Nanoparticles and Their Electrocatalytic Effect on Oxygen Reduction Reaction. Chem. Mater. 2010, 22, 4282-4294.

(11) Burr, L.; Schubert, I.; Sigle, W.; Trautmann, C.; ToimilMolares, M. E. Surface Enrichment in Au-Ag Alloy Nanowires and Investigation of the Dealloying Process. J. Phys. Chem. C 2015, 119, 20949-20956.

(12) Gell, L.; Hakkinen, H. Theoretical Analysis of the $\mathrm{M}_{12} \mathrm{Ag}_{32}(\mathrm{SR})_{40}{ }^{4-}$ and $\mathrm{XM}_{12} \mathrm{Ag}_{32}(\mathrm{SR})_{30}{ }^{4-}$ Nanoclusters $(\mathrm{M}=\mathrm{Au}$, Ag; X = H, Mn). J. Phys. Chem. C 2015, 119, 10943-10948.

(13) Vysakh, A. B.; Babu, C. L.; Vinod, C. P. Demonstration of Synergistic Catalysis in Au@Ni Bimetallic Core-Shell Nanostructures. J. Phys. Chem. C 2015, 119, 8138-8146.

(14) Strasser, P.; et al. Lattice-Strain Control of the Activity in Dealloyed Core-Shell Fuel Cell Catalysts. Nat. Chem. 2010, 2, 454460.

(15) Sun, J.; Yang, F.; Zhao, D.; Chen, C.; Yang, X. Integrated Logic Gate for Fluorescence Turn-on Detection of Histidine and Cysteine Based on $\mathrm{Ag} / \mathrm{Au}$ Bimetallic Nanoclusters-Cu ${ }^{2+}$ Ensemble. ACS Appl. Mater. Interfaces 2015, 7, 6860-6866.

(16) Markides, H.; Rotherham, M.; El Haj, A. J. Biocompatibility and Toxicity of Magnetic Nanoparticles in Regenerative Medicine. J. Nanomater. 2012, 2012, 1-11.

(17) Gao, Y.; Lim, J.; Teoh, S.-H.; Xu, C. Emerging Translational Research on Magnetic Nanoparticles for Regenerative Medicine. Chem. Soc. Rev. 2015, 44, 6306-6329.

(18) Liao, Q.; Li, N.; Jin, S.; Yang, G.; Wang, C. All-Solid-State Symmetric Supercapacitor Based on $\mathrm{Co}_{3} \mathrm{O}_{4}$ Nanoparticles on Vertically Aligned Graphene. ACS Nano 2015, 9, 5310-5317.

(19) Gandhi, V.; Ganesan, R.; Abdulrahman Syedahamed, H. H.; Thaiyan, M. Effect of Cobalt Doping on Structural, Optical, and Magnetic Properties of $\mathrm{ZnO}$ Nanoparticles Synthesized by Coprecipitation Method. J. Phys. Chem. C 2014, 118, 9715-9725.

(20) Duan, S.; Wang, R. Bimetallic Nanostructures with Magnetic and Noble Metals and their Physicochemical Applications. Prog. Nat. Sci. 2013, 23, 113-126.
(21) Ge, Q.; Huang, Y.; Qiu, F.; Li, S. Bifunctional Catalysts for Conversion of Synthesis Gas to Dimethyl Ether. Appl. Catal., A 1998, $167,23-30$.

(22) Nelli, D.; Ferrando, R. Core-Shell vs Multi-Shell Formation in Nanoalloy Evolution from Disordered Configurations. Nanoscale 2019, 11, 13040-13050.

(23) Ferrando, R. Structure and Properties of Nanoalloys; Elsevier: Amsterdam, 2016; Vol. 10.

(24) Schnedlitz, M.; Lasserus, M.; Knez, D.; Hauser, A. W.; Hofer, F.; Ernst, W. E. Thermally Induced Breakup of Metallic Nanowires: Experiment and Theory. Phys. Chem. Chem. Phys. 2017, 19, 94029408.

(25) Hauser, A. W.; Schnedlitz, M.; Ernst, W. E. A Coarse-Grained Monte-Carlo Approach to Diffusion Processes in Metallic Nanoparticles. Eur. Phys. J. D 2017, 71, 150.

(26) Lasserus, M.; Schnedlitz, M.; Knez, D.; Messner, R.; Schiffmann, A.; Lackner, F.; Hauser, A. W.; Hofer, F.; Ernst, W. E. Thermally Induced Alloying Processes in a Bimetallic System at the Nanoscale: AgAu Sub-5 nm Core-Shell Particles Studied at Atomic Resolution. Nanoscale 2018, 10, 2017-2024.

(27) Schnedlitz, M.; Lasserus, M.; Meyer, R.; Knez, D.; Hofer, F.; Ernst, W. E.; Hauser, A. W. On the Stability of Core-Shell Nanoparticles for Catalysis at Elevated Temperatures: Structural Inversion in the $\mathrm{Ni}-\mathrm{Au}$ System Observed at Atomic Resolution. Chem. Mater. 2018, 30, 1113-1120.

(28) Lasserus, M.; Knez, D.; Schnedlitz, M.; Hauser, A. W.; Hofer, F.; Ernst, W. E. On the passivation of iron particles at the nanoscale. Nanoscale Adv. 2019, 1, 2276.

(29) Schnedlitz, M.; Fernandez-Perea, R.; Knez, D.; Lasserus, M.; Schiffmann, A.; Hofer, F.; Hauser, A. W.; de Lara-Castells, M. P.; Ernst, W. E. Effects of the Core Location on the Structural Stability of $\mathrm{Ni}-\mathrm{Au}$ Core-Shell Nanoparticles. J. Phys. Chem. C 2019, 123, 20037-20043.

(30) Volk, A.; Thaler, P.; Koch, M.; Fisslthaler, E.; Grogger, W.; Ernst, W. E. High Resolution Electron Microscopy of Ag-Clusters in Crystalline and Non-crystalline Morphologies Grown Inside Superfluid Helium Nanodroplets. J. Chem. Phys. 2013, 138, 214312.

(31) Thaler, P.; Volk, A.; Knez, D.; Lackner, F.; Haberfehlner, G.; Steurer, J.; Schnedlitz, M.; Ernst, W. E. Synthesis of Nanoparticles in Helium Droplets - A Characterization Comparing Mass-Spectra and Electron Microscopy Data. J. Chem. Phys. 2015, 143, 134201.

(32) Toennies, J. P.; Vilesov, A. F. Superfluid Helium Droplets: A Uniquely Cold Nanomatrix for Molecules and Molecular Complexes. Angew. Chem., Int. Ed. 2004, 43, 2622-2648.

(33) de Lara-Castells, M. P.; Aguirre, N. F.; Stoll, H.; Mitrushchenkov, A. O.; Mateo, D.; Pi, M. Communication: Unraveling the ${ }^{4} \mathrm{He}$ droplet-mediated soft-landing from $a b$ initioassisted and time-resolved density functional simulations: $\mathrm{Au} @{ }^{4} \mathrm{He}_{300} / \mathrm{TiO}_{2}(110)$. J. Chem. Phys. 2015, 142, 131101.

(34) Aguirre, N. F.; Mateo, D.; Mitrushchenkov, A. O.; Pi, M.; de Lara-Castells, M. P. Helium Mediated Deposition: Modeling the He$\mathrm{TiO}_{2}(110)-(1 \times 1)$ Interaction Potential and Application to the Collision of a Helium Droplet from Density Functional Calculations. J. Chem. Phys. 2012, 136, 124703.

(35) Thaler, P.; Volk, A.; Ratschek, M.; Koch, M.; Ernst, W. E. Molecular Dynamics Simulation of the Deposition Process of Cold Ag-Clusters Under Different Landing Conditions. J. Chem. Phys. 2014, 140, 044326.

(36) Fernández-Perea, R.; Gómez, L. F.; Cabrillo, C.; Pi, M.; Mitrushchenkov, A. O.; Vilesov, A. F.; de Lara-Castells, M. P. Helium Droplet-Mediated Deposition and Aggregation of Nanoscale Silver Clusters on Carbon Surfaces. J. Phys. Chem. C 2017, 121, 2224822257.

(37) Plimpton, S. Fast Parallel Algorithms for Short-Range Molecular Dynamics. J. Comput. Phys. 1995, 117, 1-19.

(38) Zhou, X. W.; Johnson, R. A.; Wadley, H. N. G. Misfit-Energy Increasing Dislocations in Vapor-Deposited CoFe/ NiFe Multilayers. Phys. Rev. B: Condens. Matter Mater. Phys. 2004, 69, 10. 
(39) Kresse, G.; Furthmüller, J. Efficient Iterative Schemes for Ab initio Total-Energy Calculations Using a Plane-Wave Basis Set. Phys. Rev. B: Condens. Matter Mater. Phys. 1996, 54, 11169.

(40) Kresse, G.; Joubert, D. From Ultrasoft Pseudopotentials to the Projector Augmented-Wave Method. Phys. Rev. B: Condens. Matter Mater. Phys. 1999, 59, 1758.

(41) de Lara-Castells, M. P.; Cabrillo, C.; Micha, D. A.; Mitrushchenkov, A. O.; Vazhappilly, T. Ab Initio Design of Light Absorption Through Silver Atomic Cluster Decoration of $\mathrm{TiO}_{2}$. Phys. Chem. Chem. Phys. 2018, 20, 19110-19119.

(42) de Lara-Castells, M. P.; Hauser, A. W.; Ramallo-López, J. M.; Buceta, D.; Giovanetti, L. J.; López-Quintela, M. A.; Requejo, F. G. Increasing the Optical Response of $\mathrm{TiO}_{2}$ and Extending it into the Visible Region through Surface Activation with Highly Stable $\mathrm{Cu}_{5}$ Clusters. J. Mater. Chem. A 2019, 7, 7489-7500.

(43) Grimme, S.; Ehrlich, S.; Goerigk, L. Effect of the Damping Function in Dispersion Corrected Density Functional Theory. J. Comput. Chem. 2011, 32, 1456-1465.

(44) Grimme, S.; Antony, J.; Ehrlich, S.; Krieg, H. A Consistent and Accurate $\mathrm{Ab}$ Initio Parametrization of Density Functional Dispersion Correction (DFT-D) for the 94 Elements H-Pu. J. Chem. Phys. 2010, 132, 154104.

(45) López-Caballero, P.; Hauser, A. W.; de Lara-Castells, M. P. Exploring the Catalytic Properties of Unsupported and $\mathrm{TiO}_{2}-$ Supported $\mathrm{Cu}_{5}$ Clusters: $\mathrm{CO}_{2}$ Decomposition to $\mathrm{CO}$ and $\mathrm{CO} 2$ Photoactivation. J. Phys. Chem. C 2019, 123, 23064-23074.

(46) Perdew, J. P.; Burke, K.; Ernzerhof, M. Generalized Gradient Approximation Made Simple. Phys. Rev. Lett. 1996, 77, 3865-3868.

(47) Perdew, J. P.; Ruzsinszky, A.; Csonka, G. I.; Vydrov, O. A.; Scuseria, G. E.; Constantin, L. A.; Zhou, X.; Burke, K. Restoring the Density-Gradient Expansion for Exchange in Solids and Surfaces. Phys. Rev. Lett. 2008, 100, 136406.

(48) Perdew, J. P.; Ruzsinszky, A.; Csonka, G. I.; Vydrov, O. A.; Scuseria, G. E.; Constantin, L. A.; Zhou, X.; Burke, K. Erratum: Restoring the Density-Gradient Expansion for Exchange in Solids and Surfaces [Phys. Rev. Lett. 100, 136406 (2008)]. Phys. Rev. Lett. 2009, 102, 039902.

(49) Blöchl, P. E. Projector Augmented-Wave Method. Phys. Rev. B: Condens. Matter Mater. Phys. 1994, 50, 17953.

(50) Monkhorst, H. J.; Pack, J. D. Special Points for Brillouin-Zone Integration. Phys. Rev. B 1976, 13, 5188-5192.

(51) Söderlind, P.; Moriarty, J. A.; Wills, J. M. First-Principles Theory of Iron up to Earth-Core Pressures: Structural, Vibrational, and Elastic Properties. Phys. Rev. B: Condens. Matter Mater. Phys. 1996, 53, 14063-14072.

(52) Palomares-Baez, J.-P.; Panizon, E.; Ferrando, R. Nanoscale Effects on Phase Separation. Nano Lett. 2017, 17, 5394-5401.

(53) Baletto, F.; Mottet, C.; Ferrando, R. Growth of Three-Shell Onionlike Bimetallic Nanoparticles. Phys. Rev. Lett. 2003, 90, 135504.

(54) Knez, D.; Schnedlitz, M.; Lasserus, M.; Hauser, A. W.; Ernst, W. E.; Kothleitner, G.; Hofer, F. The impact of swift electrons on the segregation of Ni-Au nanoalloys. Appl. Phys. Lett. 2019, 115, 123103.

(55) Lai, K. C.; Han, Y.; Spurgeon, P.; Huang, W.; Thiel, P. A.; Liu, D.-J.; Evans, J. W. Reshaping, Intermixing, and Coarsening for Metallic Nanocrystals: Nonequilibrium Statistical Mechanical and Coarse-Grained Modeling. Chem. Rev. 2019, 119, 6670-6768.

(56) Medasani, B.; Haranczyk, M.; Canning, A.; Asta, M. Vacancy Formation Energies in Metals: A Comparison of MetaGGA with LDA and GGA Exchange-Correlation Functionals. Comput. Mater. Sci. 2015, 101, 96-107.

(57) Zhao, S.; Stocks, G. M.; Zhang, Y. Defect energetics of concentrated solid-solution alloys from ab initio calculations:

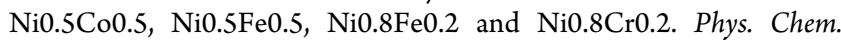
Chem. Phys. 2016, 18, 24043-24056.

(58) Eremeev, S. V.; Lipnitskii, A. G.; Potekaev, A. I.; Chulkov, E. V. Vacancies at the Surfaces of F.C.C. Metals. Russ. Phys. J. 1997, 40, 276-284.

(59) Chakrabarty, A.; Bentria, E. T.; Omotayo, S. A.; Bouhali, O.; Mousseau, N.; Becquart, C. S.; El Mellouhi, F. Elucidating the Role of
Extended Surface Defects at Fe Surfaces on CO Adsorption and Dissociation. Appl. Surf. Sci. 2019, 491, 792-798.

(60) Li, B.; Zhang, Q.; Chen, L.; Cui, P.; Pan, X. Vacancy-Mediated Diffusion of Carbon in Cobalt and its Influence on CO Activation. Phys. Chem. Chem. Phys. 2010, 12, 7848-7855.

(61) Okamoto, H.; Massalski, T.; Nishizawa, T.; Hasebe, M. The Au-Co (gold-cobalt) system. Bull. Alloy Phase Diagrams 1985, 6, $449-454$.

(62) Okamoto, H.; Massalski, T.; Swartzendruber, L.; Beck, P. The Au-Fe (gold-iron) system. Bull. Alloy Phase Diagrams 1984, 5, 592601. 